\title{
Percepción del compromiso organizacional en estudiantes de una escuela de arte de Lima
}

\section{Perception of organizational commitment in students of an art school in Lima}

\author{
Tania María Anaya Figueroa ${ }^{1}$ \\ Escuela Nacional Superior de Folklore José María Arguedas
}

Recibido: $14-03-20$

Aceptado: $12-06-20$

\section{Resumen}

La investigación tiene por objetivo conocer el compromiso organizacional en una escuela de arte de Lima Metropolitana; es un estudio descriptivo y el diseño es no experimental, transversal con enfoque cuantitativo. La muestra fue de tipo probabilístico y estuvo conformada por 201 estudiantes que cursaban estudios entre el ciclo III y IX de la carrera profesional de Docente en Educación Artística y Artista Profesional en la especialidad de música y danza. El instrumento de recolección de datos fue un cuestionario adaptado de la escala de compromiso de Allen y Meyer (1990) y Meyer y Allen (1993), el cual fue validado por juicio de expertos .97 coeficiente de V de Aiken. La confiabilidad con el coeficiente de Alfa de Cronbach fue .90 , considerada muy alta. Los resultados arrojaron que el compromiso organizacional se encuentra entre mediano y alto. A nivel de las dimensiones, la afectiva se encuentra en un nivel alto y las de continuidad y normativa están en nivel medio y alto; en la correlación entre dimensiones se ve que la dimensión afectiva muestra una relación positiva con la normativa y de continuidad.

Palabras clave: Compromiso organizacional; compromiso afectivo; compromiso de continuidad; compromiso normativo; educación superior.

\begin{abstract}
The research aims to know the organizational commitment in an art school of Metropolitan Lima; it is a descriptive study and the design is non-experimental, cross-sectional with a quantitative approach. The sample was of probabilistic type and was conformed by $201 \mathrm{stu}-$ dents who studied between the cycle III and IX of the professional career of teacher in Artistic Education and Professional Artist in the specialty of music and dance. The data collection
\end{abstract}

\footnotetext{
${ }^{1}$ Docente de la Escuela Nacional Superior de Folklore José María Arguedas. E-mail: tanaya@escuelafolklore.edu.pe - hutanafa@hotmail.com ORCID: https://orcid.org/0000-0002-5981-918X
}

(C) Los autores. Este artículo es publicado por la Revista de Investigación en Psicología de la Facultad de Psicología, Universidad Nacional Mayor de San Marcos. Este es un artículo de acceso abierto, distribuido bajo los términos de la licencia Creative Commons Atribucion - No Comercia_Compartir Igual 4.0 Internacional. (http://creativecommons.org/licenses/by-nc-sa/4.0/) que permite el uso no comercial, distribución y reproducción en cualquier medio, siempre que la obra original sea debidamente citada. 
instrument was a questionnaire adapted from the scale of commitment of Allen and Meyer (1990) and Meyer, Allen (1993), which was validated by expert judgment .97 Aiken's V coefficient. Reliability with the Cronbach's alpha coefficient was .90 , considered very high. The results showed that the organizational commitment is between medium and high. At the level of the dimensions, the affective is at a high level and those of continuity and regulations are at medium and high level; the correlation between dimensions shows that the affective dimension shows a positive relationship with the normative and continuity.

Keywords: Organizational commitment; affective commitment; continued commitment; normative commitment; higher education.

En los últimos años el interés por la calidad de la enseñanza universitaria se ha incrementado de manera sustancial. Hasta hace poco tiempo la mayoría de los trabajos empíricos sobre el aprendizaje autorregulado se habían centrado en el estudio de los escolares, pero la enseñanza en la escuela tradicional se ha caracterizado por la presencia de un fuerte control externo, en el cual el maestro asume la responsabilidad.

La educación superior ha empezado a ser analizada desde diferentes puntos de vista; mientras algunos se centran en el desarrollo académico -es decir su proyecto formativo- otros analizan diversos factores que van influyendo de manera directa o indirecta en el mismo. Por ello, es necesario que se realicen investigaciones desde diferentes ámbitos con la finalidad de tener aportes desde diversas áreas para mejorarla en su integridad. En este contexto las escuelas de arte del Perú en el año 2008 (Ley 29292) obtuvieron el rango universitario, por lo que deben cumplir las exigencias de la Ley Universitaria y también las que demandan la sociedad y los usuarios de sus servicios.

Esta investigación busca estudiar el compromiso organizacional de los estudiantes, que es una variable analizada a nivel de trabajadores desde hace varias décadas; sin embargo, ha sido una de las menos estudiadas a nivel de alumnos de educación superior. Investigar el aspecto actitudinal en los jóvenes universitarios resulta de suma importancia debido que hoy son estos aspectos los que les permiten cumplir con sus metas personales y profesionales. En los estudios revisados, desde el ámbito educativo, se ha encontrado que las investigaciones no han tomado en cuenta cómo se manifiesta la relación de los estudiantes con la institución desde el ámbito afectivo y el deseo de concluir su carrera; asimismo, la preocupación que tienen sobre el aspecto normativo. El análisis de la variable, en este trabajo, se realiza desde la psicología organizacional; por ello, se han encontrado pocos estudios similares, lo que convierte esta investigación en original y novedosa.

El estudio pone énfasis en trabajar el compromiso de los estudiantes con la organización; esta perspectiva ayudará a conocer su comportamiento de una manera diferente a la trabajada en investigaciones orientadas básicamente al estudio de categorías como: nivel de reto académico, aprendizaje colaborativo-activo, 
interacción entre estudiante y docente, experiencias educativas enriquecedoras y ambiente de apoyo universitario (Pineda y otros, 2014).

Su estudio resulta muy importante ya que sería -junto a variables como satisfacción, clima en el aula y motivación de logro- una de las que más influye en el rendimiento académico y la conclusión de los estudios, incluyendo la satisfacción con la vida en general (Gutiérrez, Tomás y Alberola, 2018). Según Frías (2014), plantea que el compromiso y la satisfacción no son variables que aseguran la permanencia de la generación Y en una institución; pues la carencia de planes de desarrollo profesional es uno de los determinantes de su continuidad y que cobra un valor especial las posibilidades de desarrollo que encuentre la persona en una institución. Por otro lado, Minaya (2014) señala que el compromiso de los docentes se relaciona positivamente con el liderazgo transformacional de los directores y está vinculado al proceso de planificación educativa, hallado que un $59.3 \%$ de los docentes registran un compromiso organizacional medio, y el $40.7 \%$ un nivel bajo.

El compromiso organizacional es definido como la fuerza relativa de la identificación de una persona con una institución (Meyer y Allen, 1984). Es una fuerza que une al individuo con una acción y es importante para un objetivo particular (Meyer y Herscovitch, 2001). En el caso de los jóvenes, consiste en la identificación que tienen con su centro de estudios. Es un factor que les ayuda a enfrentar diversas exigencias académicas, como elaborar buenos trabajos, rendir evaluaciones satisfactorias, llegar puntuales a clase, y destinar cierta cantidad de horas a cumplir los objetivos de cada asignatura, postergando actividades sociales o de otro tipo.

Para realizar esta investigación se eligió el modelo teórico multifactorial desarrollado por Allen y Meyer (1990) Meyer y Allen (1984 y 1993), en el cual se incluyen tres dimensiones: compromiso afectivo, normativo y de continuidad.

El compromiso afectivo está asociado al deseo, debido a que las personas establecen lazos emocionales con una institución (Chiang, Nuñez y Salazar, 2010). Es el grado afectivo por el cual una persona quiere permanecer en una organización, le importa lo que sucede y se esmera por ella. Es una dimensión cargada de un aspecto emocional que mueve a la persona y que se materializa en las experiencias positivas y negativas que va recibiendo (Robbins y Judge, 2015).

El compromiso normativo está asociado al deber, a la lealtad con la institución. Existe un aspecto moral y de reciprocidad por haber recibido algún beneficio y surge la necesidad de retribución. Se le considera el sentimiento de deber permanecer en la entidad (Allen y Meyer, 1990) y está influenciado por las experiencias antes de ingresar a la organización o después, cuando empieza la interrelación con las normas.

El compromiso de continuidad está asociado a la necesidad. En el caso de los estudiantes de educación superior está muy ligado a cumplir con sus metas 
profesionales. También conocida como la variable calculadora, se manifiesta cuando la persona toma conciencia de la inversión -en tiempo y esfuerzo- que ha realizado en la institución y evalúa los costos financieros, físicos, psicológicos y las expectativas (Arias, 2001).

Según Dávila y Jiménez (2014), el compromiso afectivo es el más importante para generar bienestar en las personas, el compromiso normativo solo es un predictor del afecto positivo, y el compromiso de continuidad no predice ningún aspecto de bienestar. San Martín (2013) encontró que el compromiso afectivo y el normativo determinan el compromiso de continuidad, ratificando lo señalado por Meyer y Allen en la década del 90. Pérez (2014) descubrió que los trabajadores de una universidad privada muestran mayor compromiso afectivo, normativo y de continuidad que los trabajadores de una universidad pública. Según Máynez (2016), el compromiso afectivo tiene un impacto positivo en la transferencia interna de conocimientos; por ello, es importante generar una cultura del aprendizaje que promueva el trabajo colaborativo, la discusión y la creatividad.

En este proyecto se plantearon dos objetivos:

a. Medir la percepción del compromiso organizacional en los estudiantes de una escuela de arte de Lima.

b. Identificar y correlacionar el nivel de compromiso afectivo, normativo y de continuidad de los estudiantes.

\section{MÉTODO}

\section{Tipo de estudio y diseño de investigación}

La investigación realizada fue de tipo descriptivo, se busca estudiar una variable actitudinal en los estudiantes de educación superior con la finalidad de conocer sus percepciones sobre aspectos que van a influir en la conclusión exitosa de su formación profesional. Se recogió información sobre la variable compromiso con la finalidad de incrementar conocimiento o generalizar resultados (Sánchez y Reyes, 2015). El diseño fue no experimental transversal. Se observó los hechos en su estado natural y la intervención se dio en un solo momento (Hernández, Fernández y Batista, 2010).

\section{Población y muestra}

La población estuvo conformada por 417 jóvenes de las carreras de Educación artística y Artista profesional en las menciones de Música y Danza, quienes pertenecen al ciclo regular de la Escuela Nacional Superior de Folklore José María Arguedas de Lima. La muestra estuvo constituida por 201 estudiantes que se encontraban entre el ciclo III y IX. No se consideraron a los estudiantes del ciclo I 
porque a la fecha de la intervención sólo tenían un mes de haberse incorporado a la escuela. El diseño fue probabilístico y se trabajó con todos los alumnos que se encontraban en el aula el día de la aplicación del instrumento.

El cuestionario fue aplicado de manera grupal durante las sesiones de clase, informándose a los estudiantes que sus respuestas serían confidenciales y solo serían usadas en la investigación; la mayoría de los estudiantes aceptó llenar el cuestionario. Fue aplicado por la investigadora y dos psicólogas en 16 aulas de las 24 existentes en el ciclo regular a lo largo de una semana. Contó con la autorización de la Dirección Académica y, en algunos casos, de los docentes que se encontraban dictando las clases.

La muestra estuvo conformada por jóvenes entre 18 y 29 años de edad; quienes en su mayoría pertenecen a los estratos sociales bajo y medio bajo, y un menor porcentaje de clase media; por lo que la mayoría debe estudiar y trabajar para poder solventar sus estudios superiores, en algunos casos cubrir sus necesidades básicas de alimentación, vivienda, transporte.

Es necesario indicar que la mayor parte de estos jóvenes, además de estudiar, están inmersos en alguna actividad económica ligada a brindar espectáculos a través de grupos artísticos, por lo que estarían considerados como trabajadores independientes y, en cierta medida, informales. Sin embargo, algunos de ellos logran tener éxito y dejan sus estudios por el trabajo, sobre todo cuando éste es muy demandante los fines de semana.

\section{Instrumento}

Se realizó una adaptación de la escala de compromiso organizacional de Allen y Meyer (1990) y Meyer y Allen (1993). El instrumento original está dirigido a trabajadores, por lo que cada ítem fue adaptado para el caso de estudiantes universitarios. El cuestionario consta de 24 ítems referidos a las tres dimensiones de la variable con una escala tipo Likert de 6 opciones. El instrumento adaptado fue validado por juicio de expertos, obteniendo un .97 según el coeficiente de $\mathrm{V}$ de Aiken (Porras, 2017) y luego se realizó una prueba piloto compuesta por 30 sujetos, los cuales no fueron parte de la muestra final, en la que se obtuvo un nivel de confiabilidad elevado y aceptable (Tabla 1).

\section{Tabla 1}

Confiabilidad del instrumento

\begin{tabular}{lcc}
\hline \multicolumn{1}{c}{ Dimensión / variable } & Alfa de Cronbach & N $^{\circ}$ de ítems \\
\hline Compromiso Organizacional & $\mathbf{9 1}$ & $\mathbf{2 4}$ \\
Compromiso afectivo & .85 & 8 \\
Compromiso normativo & .80 & 8 \\
Compromiso de continuidad & .83 & 8 \\
\hline
\end{tabular}

Fuente: Valores obtenidos con el programa Estadístico SPSS 22 
El nivel de confiabilidad por cada ítem (Tabla 2) estuvo entre .90 y .914, lo que indica que es un instrumento muy confiable para ser aplicado en estudiantes de una institución de educación superior.

Tabla 2

Confiabilidad por item

\begin{tabular}{llc}
\hline & Ítems & $\begin{array}{c}\text { Alfa de } \\
\text { Cronbach }\end{array}$ \\
\hline 1 & Me gustaría continuar el resto de mi carrera profesional en esta institución. &, 901 \\
2 & Siento de verdad, que cualquier problema en esta institución, es también mi problema. &, 912 \\
3 & Estudiar en esta institución significa mucho para mí. &, 903 \\
4 & En esta institución me siento como en familia. &, 906 \\
5 & Estoy orgulloso(a) de estudiar en esta institución. &, 901 \\
6 & Me siento emocionalmente unido a esta institución. &, 901 \\
7 & Me siento parte integrante de esta institución. &, 906 \\
8 & En el futuro me gustaría estar ligado a esta institución. &, 905 \\
9 & Creo que no estaría bien dejar esta institución aunque me vaya a beneficiar en el cambio. &, 902 \\
10 & Creo que debo mucho a esta institución. &, 905 \\
11 & Esta institución se merece mi lealtad. &, 905 \\
12 & Siento que conozco la normatividad vigente y que puedo concluir mi carrera. &, 905 \\
13 & Siento la obligación de tener que seguir estudiando en esta institución. &, 904 \\
14 & Me sentiría culpable si ahora dejara esta institución. &, 902 \\
15 & Siento que debo terminar la carrera pues invertí mucho aquí. &, 909 \\
16 & Creo que no podría dejar esta institución porque siento que tengo una obligación con la gente &, 900 \\
de aquí. & Si continuo en esta institución es porque en otra no tendría las mismas ventajas y beneficios &, 904 \\
17 & Aue recibo aquí. &, 907 \\
19 & Una de las desventajas de dejar esta institución es que hay pocas posibilidades de encontrar &, 900 \\
20 & Si ahora decidiera dejar esta institución muchas cosas en mi vida personal se verían interrum- \\
pidas. &, 900 \\
21 & En este momento, dejar esta institución supondría un gran costo para mí. &, 908 \\
22 & Creo que si dejara esta institución no tendría muchas opciones de encontrar otra similar. &, 902 \\
23 & Ahora mismo, estudio en esta institución más porque lo necesito que porque yo quiera. \\
24 & Continuaría en esta institución aunque tenga otro a la vista. &, 914 \\
\hline &, 904 \\
\hline
\end{tabular}

Fuente: Valores obtenidos con el programa Estadístico SPSS 22

\section{Baremos del compromiso organizacional}

Para interpretar los resultados se calcularon los valores máximos y mínimos y se establecieron tres escalas y rangos: Alto, Mediano y Bajo, de acuerdo a la Tabla 3. 


\section{Tabla 3}

Escalas y rangos del compromiso organizacional

\begin{tabular}{lc}
\hline Escala & Rangos \\
\hline Alto & $106-144$ \\
Mediano & $65-105$ \\
Bajo & $24-64$ \\
\hline
\end{tabular}

Fuente: Elaboración propia

\section{Procesamiento de datos}

Los datos fueron procesados en una hoja de cálculo (Excel). Para el procesamiento se utilizó el paquete estadístico SPSS 22 (Statistical Package for the Social Sciences), de manera especial se utilizó el estadístico Alpha de Cronbach para ver el nivel de confiabilidad de la prueba total y de cada uno de los ítems, el coeficiente de $\mathrm{V}$ de Aiken para la validación del instrumento por juicio de expertos, y el coeficiente de correlación de Pearson para ver la relación que se da entre las dimensiones de la variable.

\section{RESULTADOS}

En la Figura 1 se aprecia que el $48.8 \%$ de estudiantes percibe que tiene un compromiso mediano y el $47.8 \%$ que tiene un compromiso alto, mientras que sólo el $3.5 \%$ de los jóvenes percibe que tiene un bajo compromiso con la escuela.

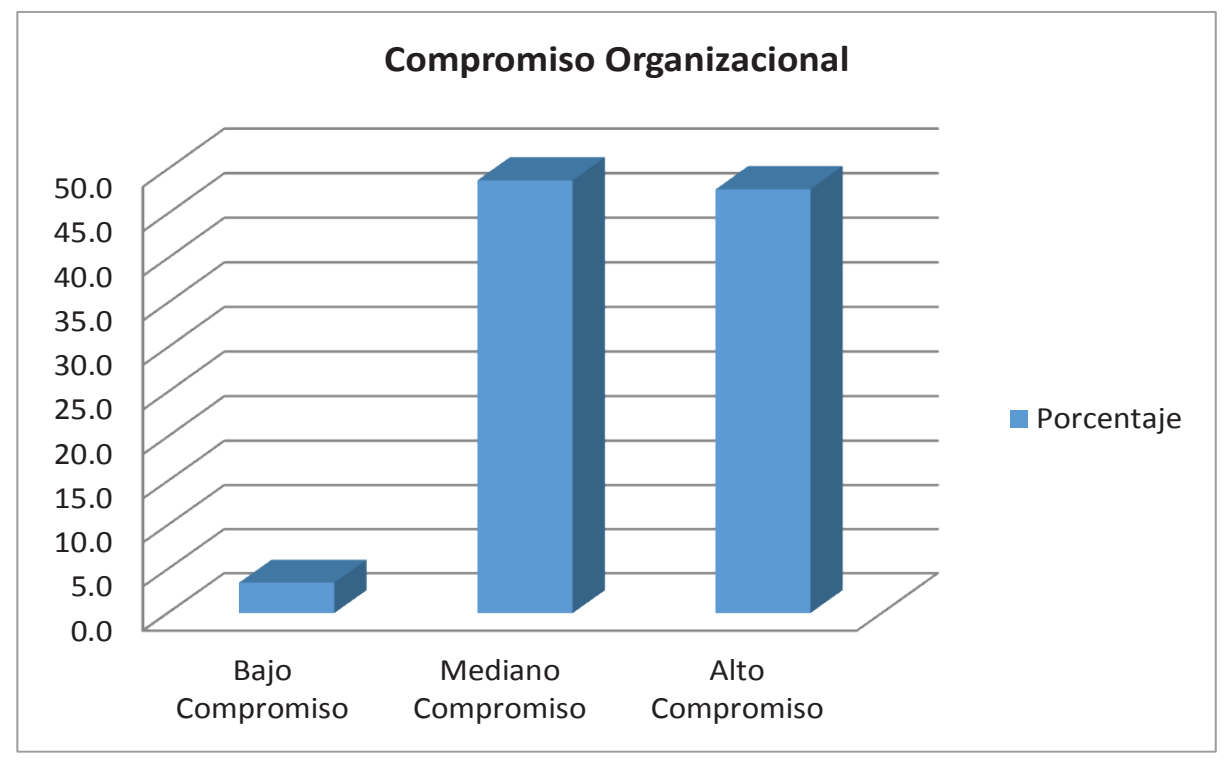

Figura 1. Porcentajes de la percepción del Compromiso Organizacional en los estudiantes 
En relación con la dimensión compromiso afectivo, el $61.2 \%$ percibe que tiene un compromiso alto, el 35.8\% mediano, y el 3\% bajo (Figura 2).

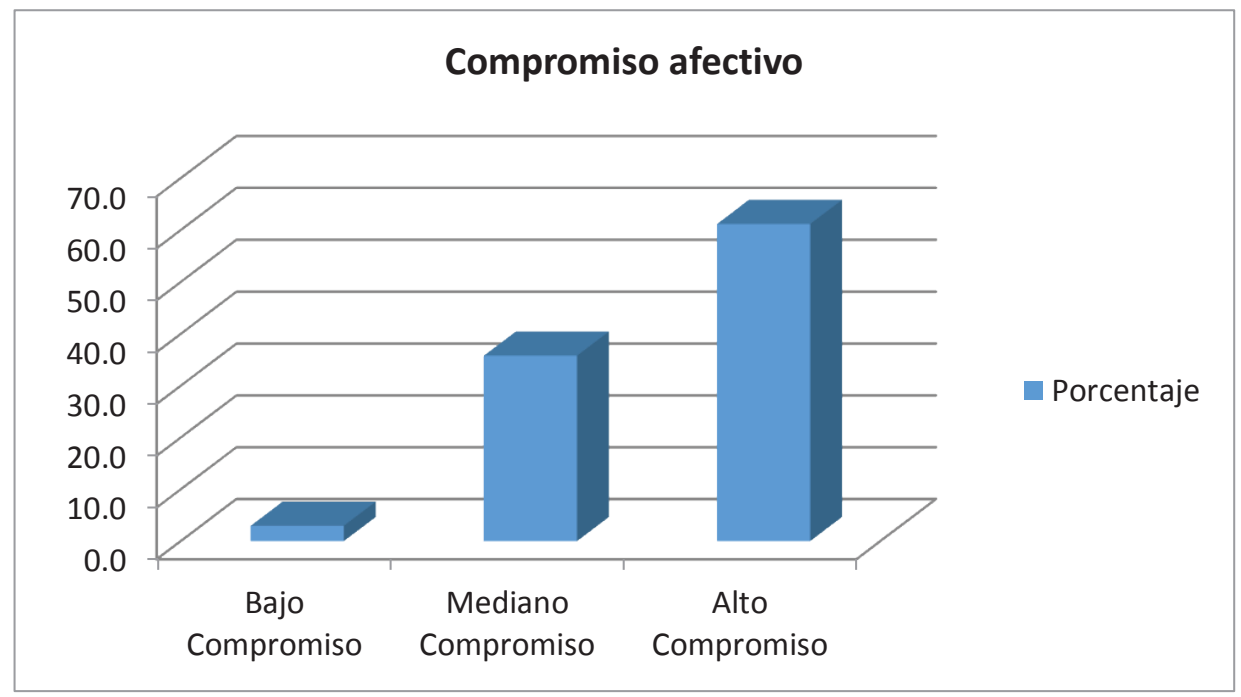

Figura 2. Porcentajes de la percepción del Compromiso Afectivo en los estudiantes

Respecto del compromiso de continuidad, un $56.7 \%$ de los estudiantes percibe que tiene un compromiso mediano, el $38.8 \%$ lo percibe alto y el $4.5 \%$ bajo (Figura 3).

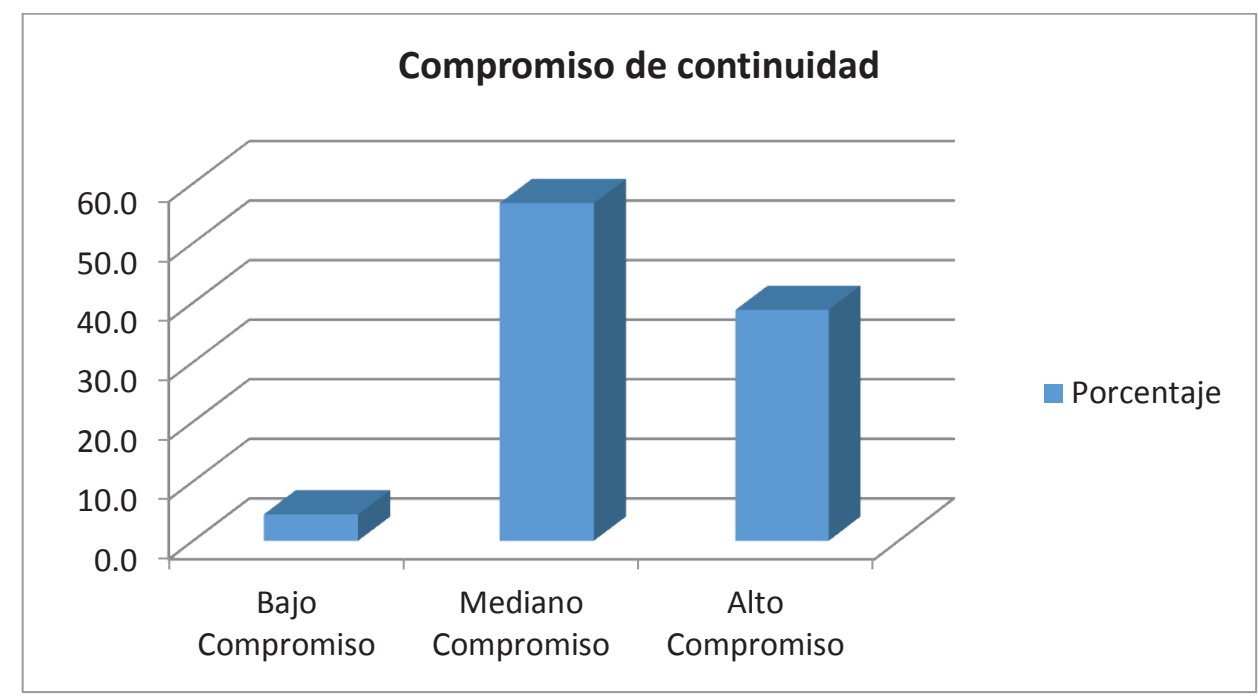

Figura 3. Porcentajes de la percepción del Compromiso de Continuidad en los estudiantes 
Sobre el compromiso normativo, la percepción de los estudiantes es de nivel mediano en un $61.2 \%$, alto en un $34.8 \%$ y bajo en un $4 \%$ (Figura 4 ),

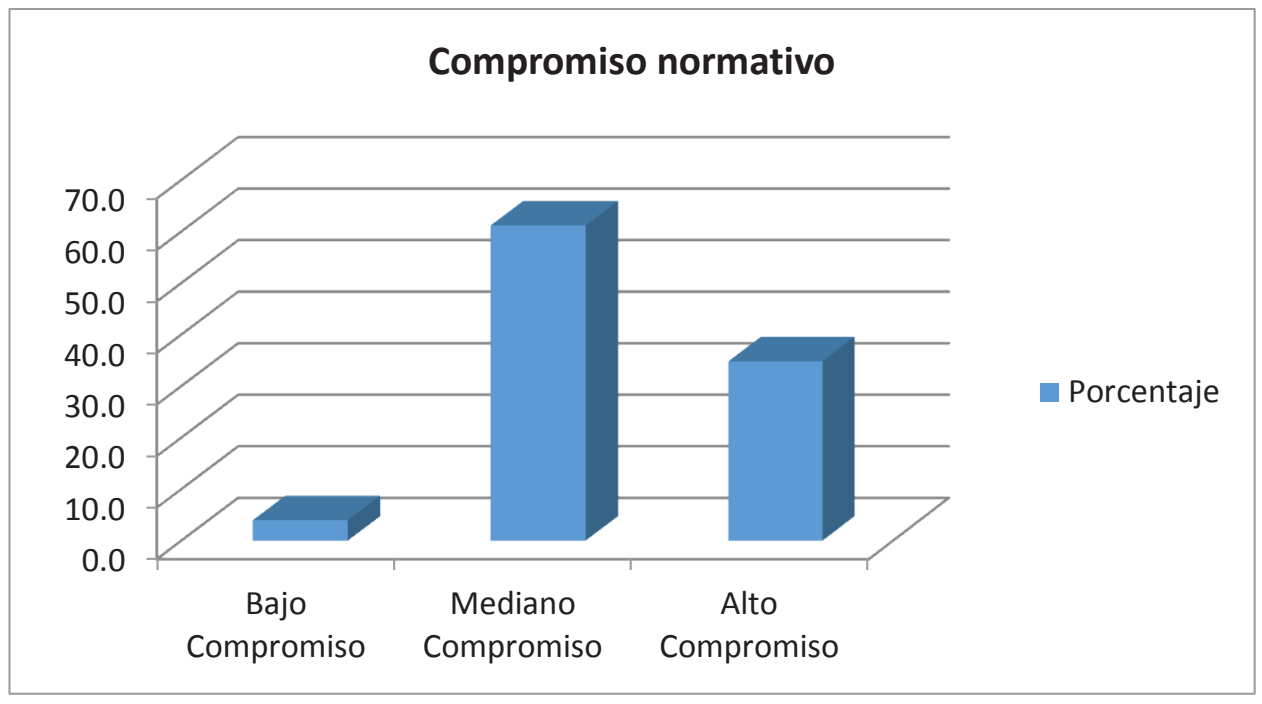

Figura 4. Porcentajes de la percepción del Compromiso Normativo en los estudiantes

En la Tabla 4 se presenta el coeficiente de correlación de Pearson y se muestra la variable con sus tres dimensiones. Se puede apreciar -siguiendo a Hernández, Fernández y Batista (2010)- que existe una correlación positiva media entre el compromiso afectivo y de continuidad; y una correlación positiva débil entre el compromiso afectivo y normativo.

\section{Tabla 4}

Coeficiente de Correlación de Pearson de las dimensiones del Compromiso Organizacional

\begin{tabular}{|c|c|c|c|c|}
\hline & & C. Afectivo & C. Normativo & C. Continuidad \\
\hline \multirow{3}{*}{ C. Afectivo } & Correlación de Pearson & 1 &, $235^{* *}$ &, $516^{* *}$ \\
\hline & Sig. (bilateral) & & 001 & 000 \\
\hline & $\mathrm{N}$ & 201 & 201 & 201 \\
\hline \multirow{3}{*}{ C. Normativo } & Correlación de Pearson &, $235^{* *}$ & 1 &, $240^{* * *}$ \\
\hline & Sig. (bilateral) & ,001 & & ,001 \\
\hline & $\mathrm{N}$ & 201 & 201 & 201 \\
\hline \multirow{3}{*}{ C. Continuidad } & Correlación de Pearson &, $516^{* *}$ &, $240^{* * *}$ & 1 \\
\hline & Sig. (bilateral) &, 000 &, 001 & \\
\hline & $\mathrm{N}$ & 201 & 201 & 201 \\
\hline
\end{tabular}

**. La correlación es significativa en el nivel 0,01 ( 2 colas). 


\section{DISCUSIÓN Y CONCLUSIONES}

Tomando como referencia la teoría multifactorial del compromiso organizacional de Meyer y Allen (1984), y en la perspectiva de la psicología positiva, se analizó el compromiso de los estudiantes de una escuela de arte vinculada a la danza y música tradicional del Perú. Se encontró que el 48.8\% de los alumnos tiene una percepción de compromiso mediano y el $47.8 \%$ alto, lo cual sugiere que la mayoría de jóvenes siente un buen nivel de compromiso organizacional con la Escuela Nacional Superior de Folklore José María Arguedas de Lima. Estos resultados son independientes de los niveles socioeconómicos de los alumnos, quienes ingresan a la institución con una alta motivación por la carrera elegida.

En la investigación realizada por Gutiérrez et al. (2018) se encontró que el compromiso académico es predeterminado en un $45 \%$ por el apoyo a la autonomía del estudiante y este factor predice, a su vez, la satisfacción con la institución. Nuestra investigación apoya este criterio. El buen nivel de compromiso organizacional encontrado en el estudio explicaría la gran cantidad de horas que los alumnos suelen dedicar en forma autónoma y voluntaria a los ensayos y prácticas de música o danza, más allá de las normas curriculares; también existe un interés personal de cada estudiante por perfeccionar su arte y mostrarlo ante un público, en diversos escenarios, incluso algunos tienen presencia en los programas de televisión vinculados al ámbito cultural. Para los estudiantes de la escuela disponer de este tipo de autonomía es un aspecto muy importante de su formación profesional. Según Loli y Cuba (2007) es necesario que los alumnos puedan experimentar y valorar los aspectos extracurriculares ofrecidos por una institución educativa.

En relación con la dimensión compromiso afectivo, el nivel es alto para el 61.2\% de estudiantes y mediano para el $35.8 \%$. Estos resultados reflejan la existencia de un elevado nivel de compromiso afectivo en la mayoría de alumnos de la escuela. Rosario y Millán (2011) han encontrado resultados similares en sus investigaciones, donde demuestran que el aspecto afectivo se convierte en una condición para lograr el compromiso organizacional. Meyer y Allen (1984) sostienen que la persona que se siente parte de una organización, es porque se siente cómoda con sus superiores, con sus compañeros de trabajo y con el trato que recibe.

En el caso de la dimensión compromiso normativo, para el 61.2\% es mediano, frente a un 34.8\% alto. Es decir, el compromiso normativo es bastante menor que el compromiso afectivo. Esto se debería, en parte, a que las escuelas de arte no cuentan con una ley específica y el ente rector -el Ministerio de Educación- no ofrece normas claras al respecto; lo que estaría perjudicando el compromiso de los alumnos a nivel normativo, pero también influyendo en aspectos como la satisfacción y el clima. Al respecto, Chiang et al. (2010) señalan que el clima organizacional tiene relación estadísticamente significativa con el compromiso afectivo y normativo. 
La dimensión compromiso de continuidad es percibida en un nivel mediano por el $56.7 \%$ de alumnos y en un nivel alto por el $38.8 \%$. Estos resultados la ubican un poco por encima de la dimensión normativa, pero muy por debajo de la dimensión afectiva. Rosario y Millán (2011) encontraron que el compromiso general y normativo son los predictores más fuertes del compromiso de continuidad. En el caso de la escuela, esta dimensión es sumamente importante porque permite que los estudiantes concluyan satisfactoriamente sus estudios y obtengan su título profesional. Al respecto, Gutiérrez et al. (2018) plantean que los docentes deben adoptar metodologías que desarrollen la motivación de los estudiantes y destaca la importancia de la tutoría académica, personal y profesional.

Sobre las correlaciones entre las tres dimensiones de la variable, se encontró -a través del coeficiente de Pearson- que existe una correlación positiva media entre el compromiso afectivo y el de continuidad (.516); y una correlación positiva débil entre el compromiso afectivo y normativo (.201) y entre el compromiso normativo y de continuidad (.240). Estos hallazgos son coincidentes con Rosario y Millán (2011), quienes sostienen que el compromiso afectivo tiene una correlación muy fuerte con el compromiso de continuidad. Sin embargo, según Gutiérrez et al (2018), el reconocimiento afectivo muchas veces no se ve reflejado al mismo nivel en el compromiso de continuidad y en el compromiso normativo.

Es probable que tener un alto compromiso organizacional ayude a los estudiantes a culminar exitosamente su carrera y pueda influir también en el compromiso de los trabajadores administrativos y docentes, generándose un efecto positivo en toda la comunidad educativa. Una investigación de Fernández (2018) con universitarios limeños encontró que si los jóvenes tienen un mayor compromiso el estrés académico es más bajo. El compromiso organizacional es un tema que debería ser analizado en todas las escuelas de arte del Perú para prevenir el problema de la deserción de alumnos. Además, es una variable importante en todos los procesos de acreditación y evaluación interna y externa. Por otro lado, sería interesante realizar una nueva investigación que busque relacionar el nivel de compromiso del estudiante con el rol de la familia y los amigos, un componente afectivo que va más allá de la institución educativa.

\section{CONCLUSIONES}

1. El $96.7 \%$ de estudiantes percibe un compromiso organizacional entre alto y mediano. Este dato explicaría la gran cantidad de horas extras de trabajo académico y artístico que los jóvenes realizan en forma voluntaria y autónoma.

2. El $61 \%$ de alumnos percibe un compromiso afectivo alto y el $35.8 \%$ mediano. Esta dimensión es una condición para lograr un buen nivel de 
compromiso general y se desarrolla a partir de actividades curriculares y extracurriculares.

3. El compromiso de continuidad es muy importante en el tiempo, ya que permite al estudiante concluir sus estudios y obtener su grado y título para insertarse en el mercado laboral. En esta dimensión el $56.7 \%$ se encuentra medianamente comprometido.

4. El compromiso normativo -se relaciona en el compromiso de continuidad- es de nivel mediano en un $61.2 \%$ y alto en un $34.8 \%$. Por ello, sería importante que el ente rector establezca normas específicas para las escuelas de arte del Perú.

5. Según la correlación de dimensiones, el compromiso afectivo es el que más se relaciona en la dimensión normativa y de continuidad. Valorar el componente afectivo de la educación es un factor clave para que la institución cumpla con éxito sus objetivos.

6. Se sugiere que la escala de compromiso organizacional para estudiantes de educación superior sea utilizada en futuras investigaciones con otras poblaciones con la finalidad comparar los resultados encontrados.

\section{Agradecimiento}

La autora agradece a la Escuela Nacional Superior de Folklore José Maria Arguedas por el apoyo a la realización de esta investigación.

\section{Fuentes de financiamiento / Funding:}

La investigación fue realizada con recursos propios.

\section{Rol de los autores / Authors Roles:}

La autora fue la responsable de realizar la investigación en sus diferentes fases así como la redacción del informe final.

\section{Aspectos éticos}

La autora se responsabiliza del contenido del artículo, el mismo que se ha elaborado respetando los derechos de autor de las fuentes, así mismo se comunica que el trabajo no ha sido presentado a ninguna otra revista para su publicación. Se ha procurado la confidencialidad de la información recogida y se solicitó la autorización a la Escuela Nacional Superior de Folklore José Maria Arguedas para la realización y publicación de este artículo.

\section{Conflicto de intereses / Competing interests:}

Como autora manifiesto no haber tenido ningún conflicto de intereses al realizar esta investigación y artículo. 


\section{REFERENCIAS}

Allen, N. y Meyer, J. (1990) The measurement and antecedents of affective, continuance and normative commitment to the organization. Journal of occupational Psycho$\log y N^{\circ}$ 63. 1-18. Great Britain: The British Psicological Society. Recuperado de doi/10.1111/j.2044-8325.1990.tb00506.x.

Arias, F. (2001) El compromiso personal hacia la organización y la intención de permanencia: algunos factores para su incremento. Revista Contaduría y Administración N²00. 5-12. Recuperado de http://www.ejournal.unam.mx/rca/200/RCA20001.pdf

Chiang, M., Nuñez, A., y Salazar, M. (2010) Compromiso del Trabajador hacia su Organización y la relación con el Clima Organizacional: Un Análisis de Género y Edad. Panorama Socioeconómico, 28(40).92-103. Recuperado de https://www.redalyc.org/ articulo.oa?id=399/39915685007

Dávila, C. y Jiménez, G. (2014) Sentido de pertenencia y compromiso organizacional: predicción del bienestar. Revista de Psicología Vol. 32 (2). 272-302. Recuperado de https://www.researchgate.net/publication/270157927

Fernández (2018) Estrés y Compromiso Académico en estudiantes de una universidad privada de Lima. (Tesis) recuperado de https://repositorioacademico.upc.edu.pe/bitstream/handle/10757/625153/Fern\%C3\%A1ndez_ca.pdf?sequence=1\&isAllowed=y

Frías, P. (2014) Compromiso y satisfacción laboral como factores de permanencia de la generación $Y$ " Universidad de Chile. (tesis) Recuperado de http://repositorio. uchile.cl/bitstream/handle/2250/117629/TESIS\%20MAGISTER\%20PFRIAS_2014. pdf? sequence $=1$

Hernández, R. Fernández, C. y Baptista, M. (2010) Metodología de la investigación. México: Mc Graw Hill.

Gutiérrez, M., Tomás, J. y Alberola, S. (2018) Apoyo docente, compromiso académico y satisfacción del alumnado universitario. Estudios sobre Educación. Vol.35. 535-555. Doi: $10.15581 / 004.34 .535-555$

Loli, A. y Cuba, E. (2007) Autoestima y compromiso organizacional en trabajadores de una universidad pública de provincias. Revista de Investigación en Psicología $N^{\circ} 10$. 103-108. UNMSM: Lima, Perú. Recuperado de Doi: https://doi.org/10.15381/rinvp. v10i1.3910

Máynez, I. (2016) Cultura y compromiso afectivo: ¿influyen sobre la transferencia interna del conocimiento? Revista de Contaduría y Administración $N^{\circ}$ 64. 666-681. México: Universidad Autónoma de Juárez. Recuperado de http://dx.doi.org/10.1016/j. cya.2016.06.003

Meyer, J. and Allen, N. (1984) Testing the "Side-Bet Theory" of Organizational Commitment: Some Methodological Considerations. Journal of Applied Psychology 1984, Vol 69. No 3. 372-378. https://scinapse.io/papers/2093181819

Meyer, J. and Allen, N. (1993): Commitment to organizations and occupations: Extension and test of the three-component conceptualization. Journal of Applied Psychology, Vol. 78. 538-551. doi.org/10.1037/0021-9010.78.4.538. 
Meyer, J. y Herscovitch, L. (2001) Commitment in the workplace: toward a general model. Human Resource Management Review Volume 11, Issue 3, Autumn 2001. 299-326. Doi: 10.1016/S1053-4822(00)00053-X.

Minaya, M. (2014) El liderazgo transformacional de los directivos y las actitudes de los docentes hacia el compromiso organizacional en la Institución Educativa $\mathrm{N}^{\mathrm{o}} 5084$ “Carlos Philips Previ” Callao 2010 - 2011. Universidad Nacional Mayor de Marcos (tesis). Recuperado de http://cybertesis.unmsm.edu.pe/bitstream/handle/cybertesis/3989/Minaya_cm.pdf?sequence $=1 \&$ isAllowed $=y$

Pérez, R. (2014) Motivación y Compromiso Organizacional en Personal Administrativo de Universidades Limeñas. PUCP (tesis). http://tesis.pucp.edu.pe/repositorio/handle/20.500.12404/5747

Pineda-Báez, Clelia; Bermúdez-Aponte, José-Javier; Rubiano-Bello, Ángela; Pava-García, Natalia; Suárez-García, Rodrigo \& Cruz-Becerra, Fabián (2014). Compromiso estudiantil y desempeño académico en el contexto universitario colombiano. Relieve, v. 20 (2), art. 3. DOI: 10.7203/relieve.20.2.4238

Porras, J. (2017) Pruebas no paramétricas usando R. Lima: Universidad Nacional Agraria La Molina.

Robbins, S. y Judge, T. (2015) Comportamiento organizacional 13 ${ }^{\text {a }}$ Edición. México: Pearson.

Rosario-Hernández, E. y Millán, Rovira, L. (2011). La relación entre las manifestaciones de conductas de ciudadanía organizacional con los cinco factores de la personalidad, satisfacción laboral, compromiso organizacional, justicia organizacional y contrato psicológico. Revista Puertorriqueña de Psicología, 22, 72-100. Recuperado de http://pepsic.bvsalud.org/scielo.php?script=sci_arttext\&pid=S194620262011000100004\&lng=pt\&tlng=es.

San Martín, F. (2013) La confianza, la satisfacción, las normas relacionales, el oportunismo y la dependencia como antecedentes del compromiso organizacional del trabajador. Revista de Contaduría y Administración $N^{\circ}$ 58. 11-38 Recuperado de http:// dx.doi.org/10.1016/S0186-1042(13)71208-7.

Sánchez, H. y Reyes, C. (2015) Metodología y diseños de la investigación científica. Lima: Editorial Bussines Suport. 